\title{
Neuroprotective Efficiency of Cyclosporine After Traumatic Brain Injury in Rats
}

\author{
Oktay GURCAN ${ }^{1}$, Merzuk Ozhan UCKUN², Ramazan Cengiz CELIKMEZ³, Omer Faruk TURKOGLU1, \\ Hakan EROGLU ${ }^{4}$, Ethem BESKONAKLI ${ }^{5}$, Levent ONER ${ }^{4}$, Yamac TASKIN ${ }^{5}$ \\ ${ }^{1}$ Ankara Ataturk Education and Research Hospital, Department of Neurosurgery, Ankara, Turkey \\ ${ }^{2}$ Ankara Numune Education and Research Hospital, Department of Neurosurgery, Ankara, Turkey \\ ${ }^{3}$ Antalya Education and Research Hospital, Department of Neurosurgery, Antalya, Turkey \\ ${ }^{4}$ Hacettepe University Faculty of Pharmacy, Depatment of Pharmaceutical Technology, Ankara, Turkey \\ ${ }^{5}$ Neurosurgeon in Private Practice, Ankara, Turkey \\ This study has been presented at the Scientific Congress of Turkish Neurosurgical Society between 20 and 24 April, 2007 at Antalya, Turkey.
}

Corresponding author: Oktay GURCAN oktaygurcan@gmail.com

\section{ABSTRACT}

\begin{abstract}
AIM: To evaluate the possible neuroprotective effects of systemic administration of cyclosporine (Cyclosporin A) after traumatic brain injury in rats.

MATERIAL and METHODS: The modified Feeney method was used as the trauma model in male Sprague Dawley rats. After the trauma, $20 \mathrm{mg} / \mathrm{kg}$ of cyclosporine was administered to the one group of the rats $(n=12)$ intraperitoneally. Twenty-four hours after injury, the subjects were sacrificed, and brain samples were removed. The level of brain edema was evaluated through the wet-dry weight method, the lipid peroxidation ratio, and histological examination by transmission electron microscopy.

RESULTS: The level of brain edema and lipid peroxidation ratio significantly decreased in the rats that received cyclosporine. Ultrastructural neurodestruction was graded, and a comparison of the scores between the experimental groups revealed significant neuroprotective effects of cyclosporine.

CONCLUSION: The results demonstrated that systemic administration of cyclosporine produces a statistically significant decrease in both the level of brain edema and lipid peroxidation ratio when compared with "no treatment". Cyclosporine, which is regularly used as an immunosuppressant agent, is also known to prevent opening of the mitochondrial permeability transition pore by unbinding mitochondrial matrix cyclophilin. Regulation of transition pore for mitochondrial permeability by cyclosporine implies that mitochondrial dysfunction following traumatic brain injury is an important event in the progressive loss of neuronal tissue.
\end{abstract}

KEYWORDS: Cyclosporine, Neuroprotection, Mitochondrial permeability transition pore, Traumatic brain injury, Rats

\section{INTRODUCTION}

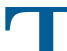
Traumatic brain injury (TBI) is a serious health problem that has great social and economic ramifications. Although the incidence of $\mathrm{TBI}$ is decreasing, $\mathrm{TBI}$ is expected to become the one of most common causes of death and disability in the near future (21). The results of the primary impact are considered irreversible, even if the impact is preventable. A group of adverse events occur as a cascade known as secondary injury, initiated just after trauma and evolving over consecutive days $(9,16,21)$. Experimental studies have revealed some of the mechanisms of secondary injury, which lead to the major neuropathological findings observed after primary injury, including alterations in endogenous excitatory amino acids and production of pro-inflammatory substances (17). The factors responsible for the destructive
Oktay GURCAN

Merzuk Ozhan UCKUN

(1) : 0000-0002-2726-0043 Omer Faruk TURKOGLU

Merzuk Ozhan UCKUN

Ramazan Cengiz CELIKMEZ
(D) : 0000-0002-3845-2665 Hakan EROGLU

(1) : 0000-0003-0547-0151 Ethem BESKONAKLI
(1) : 0000-0001-9375-1169 Levent ONER (1): 0000-0002-6510-7680 (D) : 0000-0001-6361-0074 Yamac TASKIN (1) : 0000-0003-1202-3368

(D) : 0000-0002-2903-5106 
cascade must be understood so that they can be manipulated to protect brain tissue and thus improve the outcome (22).

Neuroprotection is a growing area of research that is based mainly on pharmacological interventions aimed at preventing the secondary injury cascades and thus optimizing the outcome (21). One of the promising drugs is cyclosporine (Cyclosporin $\mathrm{A}[\mathrm{Cs} \mathrm{A}]$ ). CsA is widely used as an immunosuppressant agent after organ transplantation (2). Beside its well-known immunosuppressive activity, CsA exerts neuroprotection through calcineurin inhibition, stabilization of the mitochondrial membrane by blocking the opening of mitochondrial permeability transition ( $\mathrm{mPT}$ ) pore, and neurotrophic factor release (4). CsA is thus a therapeutic agent in as much as it blunts the effects of secondary injury and improves outcome (5).

Our aim was to investigate the neuroprotective effects of CsA after trauma. We evaluated basic parameters such as brain edema (with the wet-dry weight method), lipid peroxidation ratio, and histological findings (with the use of the Ultrastructural Neuro-destruction Grading System [UNGS] for ultrastructural findings).

\section{MATERIAL and METHODS}

\section{Materials}

The CsA was purchased from Novartis International AG (Basel, Switzerland). CsA was prepared with polyethylene glycol (MilliporeSigma, St. Louis). Anesthesia achieved with a mixture of xylazine $(10 \mathrm{mg} / \mathrm{kg})$ and ketamine $\mathrm{HCl}(90 \mathrm{mg} / \mathrm{kg})$.

\section{Animal Model}

The experimental protocol was approved by the Local Ethical Committee of Hacettepe University Ankara Approval No.: 2006/41. We used male Sprague Dawley rats, each weighing 250 to $300 \mathrm{~g}$. The animals were anesthetized with the xylazine/ketamine $\mathrm{HCl}$ mixture $(10 / 90 \mathrm{mg} / \mathrm{kg})$ intramuscularly. On a stereotactic frame, subjects were fixed, a rectal probe and a heating pad were used to control body temperatures. The rats were divided into groups $A, B, C$, and D; each group comprised 12 rats. The craniectomy was performed in all animals with the use of a high-speed drill; this was the extent of injury in rats in group $A$. The traumatic injury was achieved by the dropping of metal sterile rods weighing $24 \mathrm{~g}$ through a 9.3-cm tube over the dura (10). Rats in group $B$ received no treatment; rats in group $\mathrm{C}$ were administered only a vehicle (polyethylene glycol) so that we could investigate its possible effect. Rats in group D were administered CsA $(20 \mathrm{mg} / \mathrm{kg})$ intraperitoneally immediately after the injury. Afterwards, the rats were kept at a temperature of $25^{\circ} \mathrm{C}$ for 24 hours. Then the rats were sacrificed, and the brain tissues were removed intact 24 hours after injury (8). The contusion area was sampled and put in liquid nitrogen until analyses. The experimental groups are described in Table I.

\section{Investigation of Degree of Brain Edema}

\section{Wet-dry weight method}

The brain hemispheres of each rat were immediately weighed after the animal's sacrifice; the results were the wet weights.
The dry weights were obtained after the same hemispheres were dried at $70^{\circ} \mathrm{C}$ for 36 hours in an oven. The percentage of water content in each hemisphere was calculated as follows (19):

$\%$ Water content $=[$ (wet-dry weight $) /$ wet weight $] \times 100$

\section{Determination of Malondialdehyde Amount}

Free radicals, especially hydroxyl radicals, react with cell membranes, causing a damaging mechanism by which the unsaturated fatty acids are altered by the process of lipid peroxidation. The degree of alteration is a determinant of the reaction of membrane phospholipids with the free radicals (13).

The result of the lipid peroxidation reaction is malondialdehyde formation, theamount of which can be quantitatively determined through the thiobarbituric acid reaction. The lipid peroxidation per gram of wet tissue in nanomoles is calculated by the measurement of the color that malondialdehyde produces in the reaction with thiobarbituric acid, as demonstrated by a spectrophotometer (Shimadzu UV 120-2) at a setting of $\lambda=$ $532 \mathrm{~nm}(20)$.

\section{Ultrastructural Neuro-Destruction Grading System}

The samples were obtained from the cortical brain region as cubes measuring approximately $3 \times 3 \times 3 \mathrm{~mm}$. The cubes were fixed with gluteraldehyde solution for 24 hours immediately after the removal of tissues. Ultra-thin sections were obtained with an LKB-Nova ultramicrotome and were stained with uranyl acetate and lead citrate. We used the UNGS to quantify the ultrastructural findings; the scores are summarized in Table II.

\section{RESULTS}

\section{Wet-Dry Weight Method}

Statistical analysis was performed with the Mann-Whitney $U$ test at a significance level of 0.05 . For the animals that received intraperitoneal $20 \mathrm{mg} / \mathrm{kg}$ of CsA (group D), the water content ratios were significantly lower than those for the control group (group B), in which trauma was applied but no treatment was administered $(p<0.05)$. In the rats in group $C$ (intraperitoneal polyethylene glycol after trauma), the wet-dry tissue weights demonstrated no significant decrease in comparison with the results in group $B(p>0.05)$. The results are summarized in Figure 1.

\section{Determination of Malondialdehyde Amount}

To validate the experimental methods, malondialdehyde amounts were determined as nanomoles per gram of wet tissue for the quantification of lipid peroxidation ratio. The results of the uninjured control group (Group A) were compared with those of the injured control group (Group B) for the validation of the experimental trauma model and surgical procedures. The results clearly showed that the formation of brain edema did not occur with the craniectomy alone $(p<0.05)$. A comparison of the results for group $D$ with those of groups $B$ and $C$ revealed a significant difference in 
Table I: Experimental Groups

\begin{tabular}{lccc}
\hline Group & Craniectomy & Trauma & Treatment \\
\hline A & + & - & - \\
\hline B & + & + & i.p. Polyethylene Glycol (PEG) \\
\hline C & + & + & i.p. CsA (within PEG) \\
\hline D & + & + & - \\
\hline
\end{tabular}

PEG: Polyethylene glycol, CsA: Cyclosporine A, i.p.: intraperitoneal.

Table II: Scores Obtained with the Ultrastructural Neuro-Destruction Grading System

\begin{tabular}{ccc}
\hline Mitochondria (M) & Score & Normal \\
\hline 0 & Significant crystal \\
\hline 2 & Swelling \\
\hline & 1 & Amorphous accumulation \\
\hline
\end{tabular}

\section{Myelinated Axons}

(High Dimension)

$\begin{array}{cc}\text { Score } & \\ 0 & \text { Normal } \\ 1 & \text { Separation in the myelin layers } \\ 2 & \text { Destruction in the myelin layers } \\ 3 & \text { Honeycomb appearance }\end{array}$

\section{Myelinated Axons (Medium Dimension) Score}

\begin{tabular}{ccc}
\hline & 0 & Normal \\
\hline 1 & Separation in the myelin layers \\
\hline 2 & Destruction in the myelin layers \\
\hline
\end{tabular}

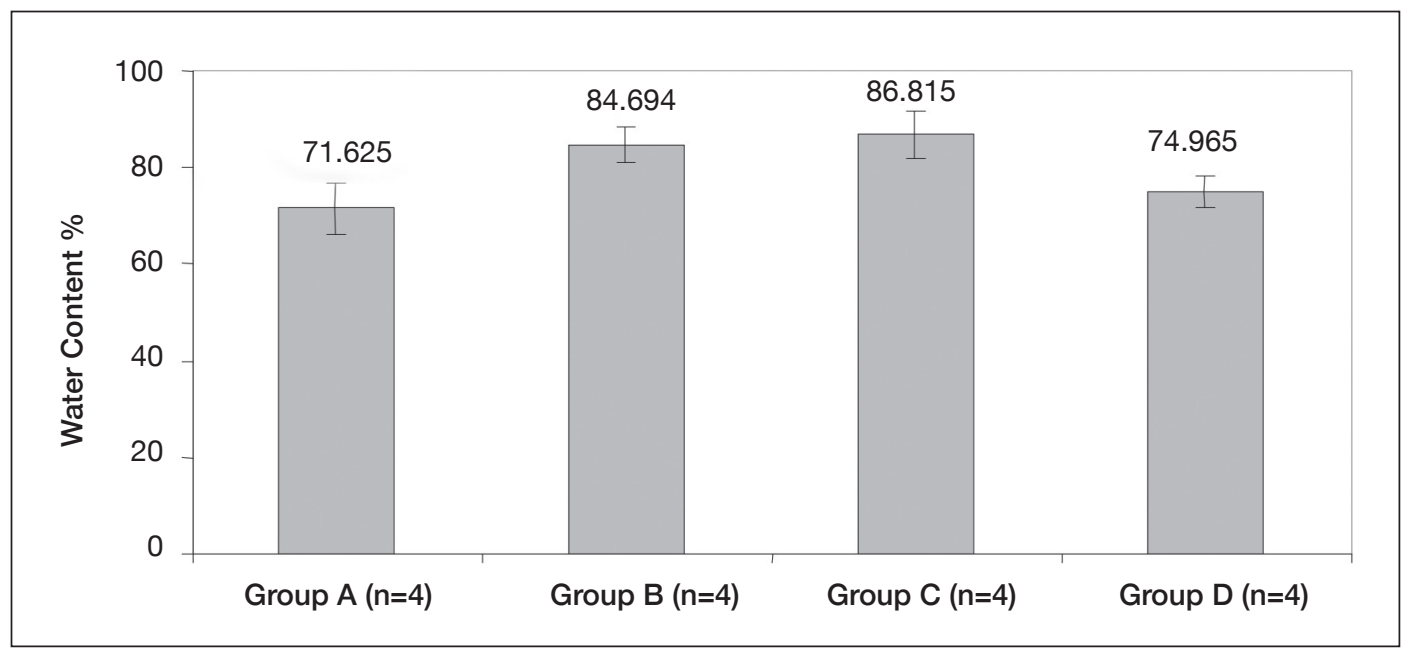

Figure 1: Water content ratios in the experimental groups (Group A: only craniectomy;

Group B: traumatic injury after craniectomy but no treatment;

Group C: traumatic injury after craniectomy, followed by administration of the vehicle;

Group D: traumatic injury after craniectomy, followed by intraperitoneal administration of cyclosporine [Cs-A]). 
lipid peroxidation ratios $(p<0.05, n=6)$. The results are shown in Figure 2.

\section{Ultrastructural Neuro-destruction Grading System}

The UNGS scoring system was used to quantify the data obtained from the microscopic evaluations. The parameters investigated and the general scores for all groups are briefly summarized in Table III.

The Mann-Whitney $U$ test was used to compare all UNGS scores in each of the investigated parameters. Results of the test showed that group D's scores were significantly different from those of group $B(p<0.05, n=2)$.

Electromicroscopic ultrastructural samples from groups $B$ and $\mathrm{D}$ are shown in Figures 3 and 4 .

\section{DISCUSSION}

TBI is a medical and social problem with high mortality and morbidity rates in adults and accounts directly for nearly $30 \%$ of deaths from trauma $(9,22)$. There are two main categories of damage after a head injury: primary and secondary. Primary injury appears immediately after injury, and secondary injury is triggered by a cascade of adverse events that start just after injury $(7,18)$. Thus primary injury is exacerbated by secondary injury, which causes neurological dysfunction (17). The secondary injury appears to be associated with posttraumatic neurochemical alterations that can both exert direct pathogenic effects on cerebral metabolism and ion homeostasis, and have neurotoxic effects. These cellular and molecular changes may include changes in the synthesis and release of neuroprotective, autodestructive, or neurotoxic cascades (7).

After TBI, decreases in levels of cellular adenosine triphosphate (ATP) and increased oxidative stress are considered to be the major causes of cell death (3). In addition, increases in glutamate level cause overactivation of $\mathrm{N}$-methyl-D-aspartate (NMDA) receptors, which in turn causes a disproportionate influx of calcium $\left(\mathrm{Ca}^{+2}\right)$ into the cell matrix that is detrimental and has been found to result neuronal loss $(3,17)$.

The increase in intracellular $\mathrm{Ca}^{+2}$ can be buffered by activation of cell membrane ion pumps and also by cellular organelles such as the endoplasmic reticulum and, mostly, by mitochondria. Mitochondria are considered pivotal organelles in the maintenance and management of intracellular $\mathrm{Ca}^{+2}$, specifically by stocking $\mathrm{Ca}^{+2}$ through several mechanisms (3).

The obvious escalation of $\mathrm{Ca}^{+2}$ in cell after glutamate exposure triggers a couple of reactions of cell damage, such as increases in free radical production and disturbances in the bioenergetic

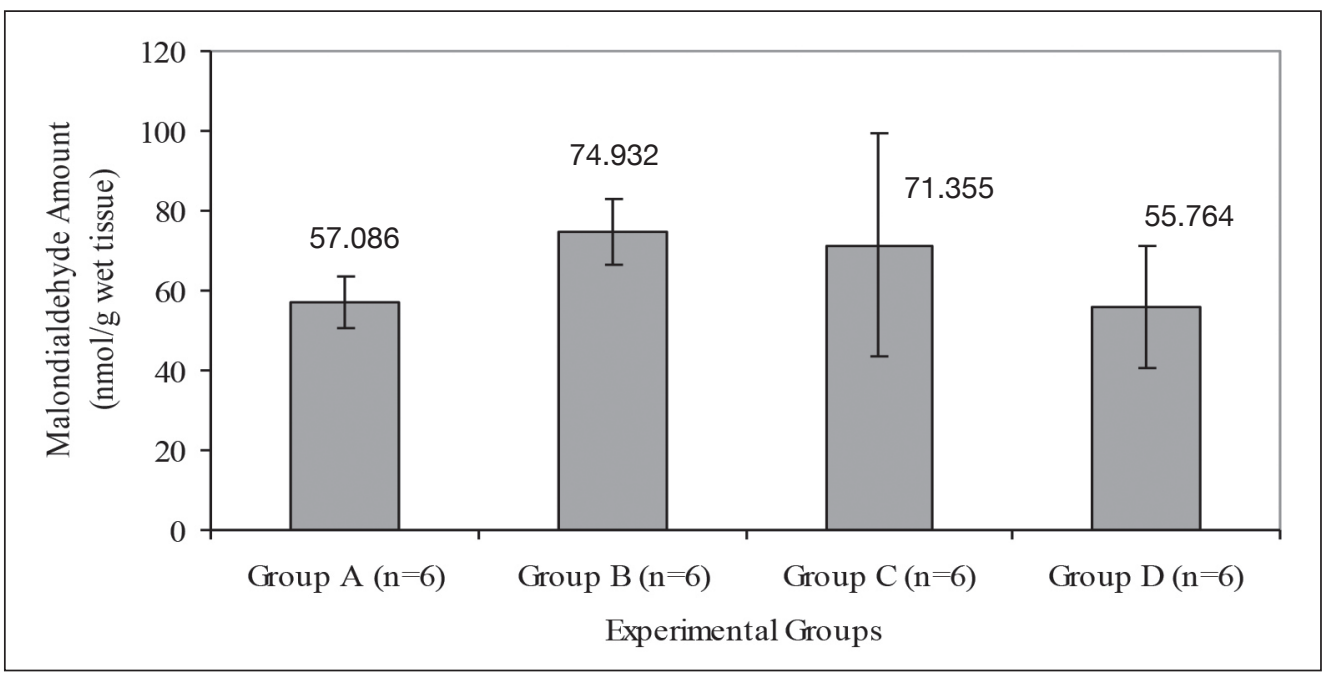

Figure 2: Lipid peroxidation ratios, expressed in terms of malondialdehyde amounts (Group A: only craniectomy; Group B: traumatic injury after craniectomy but no treatment; Group C: traumatic injury after craniectomy, followed by administration of the vehicle; Group D: traumatic injury after craniectomy, followed by intraperitoneal administration of cyclosporine [Cs-A]).

Table III: Investigational Parameters and Scores of the Experimental Groups

\begin{tabular}{lcccc}
\hline \multirow{2}{*}{ Parameter } & \multicolumn{3}{c}{ Group } \\
\cline { 2 - 5 } & A & B & C & D \\
\hline Mitochondria 1 & 11 & 31 & 29 & 23 \\
\hline Mitochondria 2 & 10 & 30 & 27 & 21 \\
\hline Large Sized Myelinated Axons 1 & 8 & 39 & 37 & 34 \\
\hline Large Sized Myelinated Axons 2 & 6 & 38 & 39 & 31 \\
\hline Medium Sized Myelinated Axons 1 & 0 & 29 & 20 & 12 \\
\hline Medium Sized Myelinated Axons 2 & 1 & 19 & 23 & 10 \\
\hline
\end{tabular}




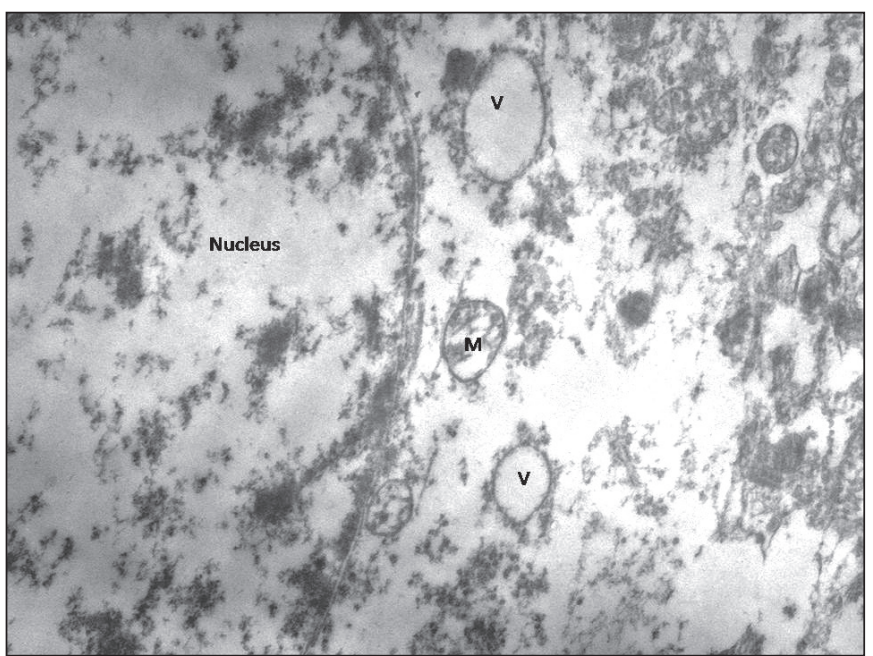

Figure 3: Electron microscopic photo of samples from group B. (M: mitochondria; V: vacuole).

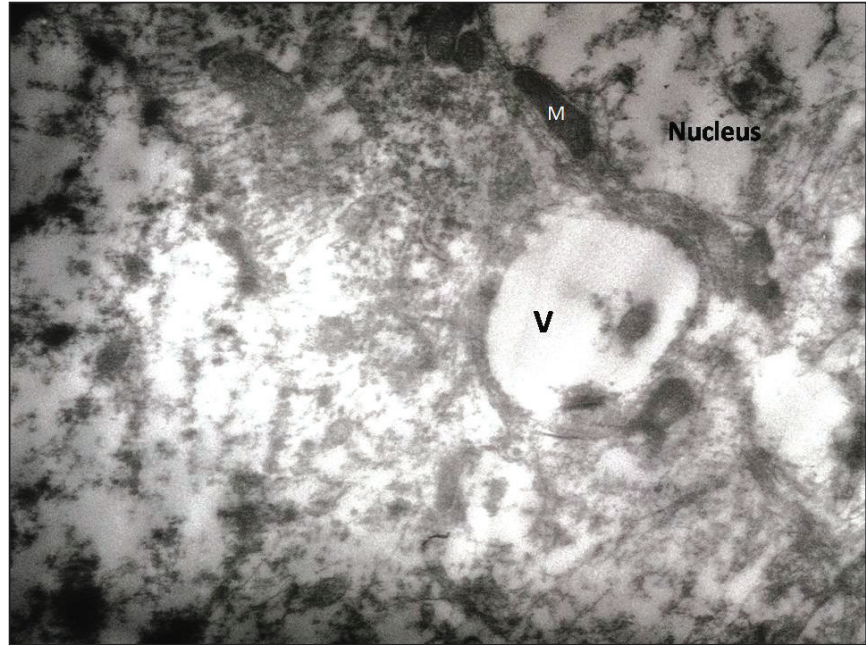

Figure 4: Electron microscopic photo of samples from group D.

capabilities of mitochondria. Overproduction of oxygen free radicals is linked to the boost in $\mathrm{Ca}^{+2}$ levels in mitochondria; however, $\mathrm{Ca}^{+2}$ can cause mitochondrial malfunction in cell death after traumatic injury $(3,17)$.

Destruction of mitochondria by $\mathrm{Ca}^{+2}$ results in disequilibrium in mitochondrial homeostasis; the failure in mitochondrial membrane potential causes an escalation in free radical formation and in diminishing ATP levels. Mitochondrial destruction is manifested by swelling and the accumulation of amorphous deposits, which causes cessation of neuron function (17). Opening of the MPT pore is proposed to be a principal reaction in the advancement of neuropathological crisis after TBI $(3,6,12)$.

CsA, is widely used for immunosuppression but has also displayed neuroprotection after TBI. It could be used to inhibit neurotoxicity by NMDA overproduction, but it also dramatically reduces $\mathrm{Ca}^{+2}$-triggered cytoskeletal changes and axon loss $(1,11,14)$.
It has been recently established that neuroprotection occurs via different pathways, including inhibition of calcineurin, closure of the MPT pore or restricting the opening of the MPT pore by inhibiting binding of mitochondrial matrix cyclophilin from the pore, and release of neurotropic factors $(3,15)$. The interaction of CsA with matrix cyclophilin results in the inhibition of the opening of the MPT pore. CsA also increases mitochondrial membrane homeostasis, which ensures the membrane potential and is essential for life. It enhances neuronal viability by inhibiting the MTP pore, thus ensuring mitochondrial membrane potential, and restores the ATP levels for the cell activity (11).

The studies cited in the preceding paragraph demonstrated that the neuroprotection achieved by CsA is dose-dependent and that the therapeutic window after traumatic injury is as long as 24 hours (15).

Sullivan and colleagues concluded that $20 \mathrm{mg} / \mathrm{kg}$ of CsA administered intraperitoneally is the most neuroprotective therapy (17-20), whereas $10 \mathrm{mg} / \mathrm{kg}$ intravenous CsA was most effective in the studies of Okonkwo and colleagues $(13,14)$. In our study, we administered $20 \mathrm{mg} / \mathrm{kg}$ (17).

For analyzing the effects of CsA on brain edema formation after TBI, we used the wet-dry weight method, as stated previously. Statistical interpretation of the findings were evaluated with the Mann-Whitney $U$ test at a significance level of less than 0.05 . For the animals that received CsA, the water content was significantly lower than the that of the control groups $(p<0.05)$.

The free radicals react with the membrane phospholipids after $\mathrm{TBI}$, and the reaction yields the final product, malondialdehyde. Malondialdehyde is known to be responsible for cell membrane destabilization, which causes cell death. In our study, we used the malondialdehyde reaction to evaluate lipid peroxidation ratios, which clarified the degree of brain edema. The MannWhitney $U$ test helps evaluate the difference between the experimental groups. The results for the animals that received systemic treatment with CsA (group D) were compared with those for both control groups (groups A and B). The difference between the results of group $D$ and those of group $B$ was statistically significant $(p<0.05)$; on the other hand, the results of the two control groups, in which no treatment was given, were not statistically different $(p>0.05)$.

The elevation in calcium levels after TBI results in mitochondrial degeneration. During the quantification, both mitochondria deformation and myelinated axon deformation were noted in more than 100 electron microscopic investigations of mitochondria. The UNGS is used for the scoring of these investigations, and it enabled us to evaluate the statistical difference between the group that received CsA after TBI (group D) and the group that received no treatment after traumatic injury (group B). By using the UNGS for scoring, the electron microscopic investigations could yield new insights into posttraumatic mitochondrial malfunctions such as the opening of the MPT pore, caused by the increase in intracellular $\mathrm{Ca}^{+2}$, which destroys mitochondrial homeostasis. 


\section{CONCLUSION}

Because $\mathrm{TBI}$ is one of the major public health problem worldwide, newer pharmacological agents must be developed to provide neuroprotection after TBI. In our study, CsA, when administered after traumatic injury, showed promise as a neuroprotective drug by maintaining calcium homeostasis and restoring the energy that is fundamental for the cell viability. Further work is necessary to evaluate the clinical usefulness of CsA.

\section{ACKNOWLEDGMENT}

The authors'd like to thank Prof. Ebru Bodur, M.D., Ph. D. from Hacettepe University School of Medicine, Department of Biochemistry for biochemical analysis, and Prof. F. Mustafa Sargon, M.D., Ph. D., Hacettepe University School of Medicine, Department of Anatomy, for assistance with the Ultrastructural Neurodestruction Grading System.

Preparation for publication of this article is partly supported by Turkish Neurosurgical Society.

\section{REFERENCES}

1. Ankarcrona M, Dypbukt JM, Bonfoco E, Zhivotovsky B, Orrenius S, Lipton SA, Nicotera P: Glutamate induced neuronal death: A succession of necrosis or apoptosis depending on mitochondrial function. Neuron 15:961-973,1995

2. Borlongan CV, Yu G, Matsukawa N, Xu L, Hess DC, Sanberg $P R$, Wang Y: Acute functional effects of cyclosporine-A and methylprednisolone treatment in adult rats exposed to transient ischemic stroke. Life Sciences 76: 1503-1512, 2005

3. Diaz-Ruiz A, Rios C, Duarte I, Correa D, Guizar-Sahagun G, Grijalva I, Ibarra A: Cyclosporin-A inhibits lipid peroxidation after spinal cordinjury in rats. Neurosci Lett 266:61-64,1999

4. Domanska-Janika K, Buzanska L, Dluzniewska J, Kozlowska $H$, Sarnowska A, Zablocka B: Neuroprotection by cyclosporin A following transient brain ischemia correlates with the inhibition of the early efflux of cytochrome $C$ to cytoplasm. Mol Brain Res 121:50-59, 2004

5. Engelborghs K, Verlooy J, Van Reempts J, Van Deuren B, Van De Ven M, Borgers M: Temporal changes in intracranial pressure in a modified experimental model of closed head injury. J Neurosurg 89:796-806, 1998

6. Eroglu H, Kas H, Oner L, Turkoglu OF, Akalan N, Sargon MF, Ozer N: The in-vitro and in-vivo characterization of PLGA: L-PLA microspheres containing dexamethasone sodium phosphate. J Microencapsul 18:603-612, 2001

7. Feeney DM, Boyeson MG, Linn RT, Murray HM, Dail WG: Responses to cortical injury: i. Methodology and local effects of contusions in the rat. Brain Res 211:67-67, 1981
8. Graham DI, Mcintosh TK, Maxwell WL, Nicoll JAR: Recent advances in neurotrauma. J Neuropathol and Exp Neurology 59: 641-651, 2000

9. Guha A: Management of traumatic brain injury: Some current evidence and applications. Postgrad Med J 80:650-653, 2004

10. Laurer HL, Lenzlinger PM, McIntosh TK: Models of traumatic brain injury. Eur J Trauma 26:95-100, 2000

11. Maxwell RE, Long DM, French LA: The effects of glucosteroids on experimental cold-induced brain edema gross morphological alterations and vascular permeability changes. J Neurosurg 34:477-487, 1971

12. Murakami K, Kondo T, Yang G, Chen SF, Morita-Fujimura Y, Chan PH: Cold injury in mice: A model to study mechanisms of brain edema and neuronal apoptosis. Prog Neurobiol 57:289-299, 1999

13. Okonkwo DO, Melon DE, Pellicane AJ, Mutlu LK, Rubin DG, Stone JR, Helm GA: Dose response of cyclosporin a in attenuating traumatic axonal injury in rat. Neuropharm and Neurotox 14:463-466, 2003

14. Okonkwo DO, Povlishock JT: An intrathecal bolus of cyclosporin A before injury preserves mitochondrial integrity and attenuates axonal disruption in traumatic brain injury. $J$ Cereb Blood Flow Metab 19:443-451, 1999

15. Schinder AF, Olson EC, Spitzer NC, Montal M: Mitochondrial dysfunction is a primary event in glutamate neurotoxicity. J Neurosci 16:6125-6133, 1996

16. Stelmasiak Z, Dudkowska-Konopa A, Rejdak K: Head trauma and neuroprotection. Med Sci Monit 6:426-432, 2000

17. Sullivan PG, Rabchevsky AG, Hicks RR, Gibson TR, FletcherTurner A, Scheff SW: Dose-response curve and optimal dosing regimen of cyclosporin $A$ after traumatic brain injury in rats. Neuroscience 101:289-295, 2000

18. Sullivan PG, Rabchevsky AG, Waldmeier PC, Springer JE: Mitochondrial permeability transition in CNS trauma: Cause or effect of neuronal cell death? J Neuroscience Research 79:231-239, 2005

19. Sullivan PG, Thompson M, Scheff SW: Continuous infusion of cyclosporin A postinjury significantly ameliorates cortical damage following traumatic brain injury. Experimental Neurology 161:631-637, 2000

20. Sullivan PG, Thompson MB, Scheff SW: Cyclosporin A attenuates acute mitochondrial dysfunction following traumatic brain injury. Exp Neurol 160: 226-234,1999

21. Tolias CM, Bullock MR: Critical appraisal of neuroprotection trials in head injury: What have we learned? Neurotherapeutics 1: $71-79,2004$

22. Yamaguchi T, Miyata $K$, Shibasaki F, Isshiki A, Uchino $H$ : Effect of cyclosporin A on immediate early gene in rat global ischemia and its neuroprotection. J Pharmacol Sci 100:73-81, 2006 